DIVISION OF THE HUMANITIES AND SOCIAL SCIENCES

CALIFORNIA INSTITUTE OF TECHNOLOGY

PASADENA, CALIFORNIA 91125

PURE STRATEGY NASH EQUILIBRIA IN NON-ZERO SUM COLONEL BLOTTO GAMES

Rafael Hortala-Vallve

London School of Economics

Aniol Llorente-Saguer

California Institute of Technology

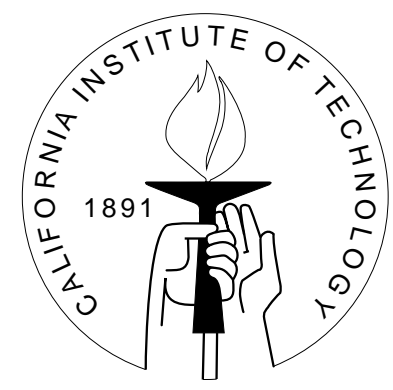

SOCIAL SCIENCE WORKING PAPER 1335

May 2010 


\title{
Pure strategy Nash equilibria in non-zero sum colonel Blotto games*
}

\author{
Rafael Hortala-Vallve \\ London School of Economics
}

\author{
Aniol Llorente-Saguer \\ Caltech
}

May 2010

\begin{abstract}
We analyze a Colonel Blotto game in which opposing parties have differing relative intensities (i.e. the game is non-zero sum). We characterize the colonels' payoffs that sustain a pure strategy equilibrium and present an algorithm that reaches the equilibrium actions (when they exist). Finally we show that the set of games with a pure strategy equilibria is non-empty.
\end{abstract}

JEL Classification: C72, D7, P16

\section{Introduction}

The Colonel Blotto game was first proposed by Borel (1921). In such a game, two colonels fight over a number of battlefields and must simultaneously divide their forces among the various battlefields. A battlefield is won by the one with the most troops and the winner is the colonel that wins the most battlefields. The game was initially studied by Borel (1921), Borel and Ville (1938) and Gross and Wagner (1950). It follows immediately from the formulation of the game that there (generally) is no pure strategy equilibrium. Recently, Roberson (2006) has fully characterized the mixed strategy equilibria when troops are perfectly divisible and Hart (2008) has done likewise when the action space is discrete.

Despite the variety of formulations of the game (discrete versus continuous forces, equal or unequal forces), the Colonel Blotto game is a zero-sum game where all regions are equally valued by both colonels, and a gain by one colonel means a loss of equal size

${ }^{*}$ We thank Antonio Cabrales and Antoni Rubi for helpful comments. 
for the other colonel. ${ }^{1}$ In this paper we analyze the game in which opposing parties have differing relative intensities. In contrast with the classical example, strict Pareto improvements may now exist: a colonel may accept losing a battlefield if that implies winning a battlefield that is of more value to him. By allowing differing relative intensities, we depart from the zero-sum nature of the game and characterize the sets of payoffs that support the existence of a pure strategy Nash equilibrium when both colonels are endowed with an equal number of indivisible troops. We prove that there can be at most a single pure strategy equilibrium and provide a simple algorithm that reaches the pure strategy equilibrium actions (whenever they exist). Finally, we show that the set of games with pure strategy equilibrium is non empty.

Our work relates to a burgeoning literature on voting and conflict resolution that proposes a new mechanism that allows agents to extract gains from the inherent heterogeneity in their preferences (see for instance Casella (2005), Jackson and Sonnenschein (2007), and Hortala-Vallve (2007)).

The rest of the paper is organized as follows. Section 2 presents the game. Section 3.1 characterizes the set of voting profiles which can constitute an equilibrium, Section 3.2 introduces an algorithm that reaches equilibrium whenever this exists and Section 3.3 describe the games which have equilibrium in pure strategies. Section 4 concludes.

\section{The model}

It's wartime. Two colonels, each on command of $T$ troops are fighting for the control of $N$ separate battlefields. They both know that the one that deploys most troops in a battlefield wins that battlefield. We want to characterise the optimal deployment of troops.

Colonels are denoted $i \in\{1,2\}$ and battlefields are denoted $n \in\{1,2, \ldots N\}$. Colonel $i$ 's payoff from winning battlefield $n$ is denoted $\theta_{n}^{i}>0$; when he loses battlefield $n$ his payoff is 0 . The payoff vector of colonel $i$ is denoted $\theta^{i}=\left(\theta_{1}^{i}, \ldots, \theta_{N}^{i}\right) \in \Theta \subseteq \mathbb{R}^{N}$. The total war payoff for each colonel is the sum of the individual payoffs across the $N$ battlefields. $^{2}$

The set of actions for each colonel is the collection of deployment profiles:

$$
\mathcal{T}:=\left\{\left(t_{1}, \ldots, t_{N}\right) \in\{0,1, \ldots, T\}^{N}: t_{1}+\ldots+t_{N}=T\right\}
$$

\footnotetext{
${ }^{1}$ There are two exceptions in which the nature of the game is non-zero sum. Kvasov (2006) characterizes the equilibrium when the allocation of forces is costly and both colonels have exactly the same number of troops. Roberson and Kvasov (2008) extend the analysis to cases in which the colonels' number of troops differ.

${ }^{2}$ Implicit in this definition of payoffs is the assumption that valuations are independent across battlefields. That is, there are no complementarities between them. If this assumption holds, results can be extended to any linear transformation of the payoffs.
} 
The winner in each battlefield is the colonel that deploys the most troops. We assume that ties (when both colonels deploy the same number of troops) are broken with the toss of a fair coin. That is,

$$
\left\{\begin{aligned}
t_{n}^{1}>t_{n}^{2} & \Rightarrow \text { colonel } 1 \text { wins battlefield } n \\
t_{n}^{1}<t_{n}^{2} & \Rightarrow \text { colonel } 2 \text { wins battlefield } n \\
t_{n}^{1}=t_{n}^{2} & \Rightarrow \text { each colonel wins battlefield } n \text { with probability } \frac{1}{2}
\end{aligned}\right.
$$

\section{Games with pure strategy equilibria}

We want to characterize the set of games that have (at least one) pure strategy equilibrium when there are more than two battlefields $(N>2)$ and a strictly positive number of troops $(T>0) .{ }^{3}$ Our argument follows three steps. Firstly, we determine which deployment profiles can constitute an equilibrium. Secondly, we describe an algorithm that reaches the pure strategy equilibrium (when this one exists). Finally, we characterize the set of payoffs that support the existence of a pure strategy equilibrium and show that this equilibrium is unique.

\subsection{Equilibrium actions}

Our first step towards characterizing the non zero-sum Colonel Blotto games which have a pure strategy equilibrium relies on distinguishing the set actions that can be part of an equilibrium. The following definitions anchor two ideas that are key in our analysis.

Definition 1 Given both colonels' deployment of troops,

- the troops of colonel $i$ in battlefield $n, t_{n}^{i}$, are decisive when deploying less troops implies a different outcome in such battlefield ${ }^{4}$

- there is a positive tie in battlefield $n$ when $t_{n}^{i}=t_{n}^{j}$ and $t_{n}^{i}>0$

The following Lemma establishes that there cannot be non decisive armies in a battlefield when ties occur in (at least) one battlefield. If this is not the case, one of the colonels has a profitable deviation by deploying non decisive troops in the battlefield where ties occur. These extra resources undo the tie and ensure a further victory for the deviating colonel.

\footnotetext{
${ }^{3}$ The cases with less than three battlefields are trivial. When $N=1$, all troops are deployed in the unique battlefield and ties occur. When $N=2$, colonels deploy all their troops in the battlefield that yields highest payoff: when the colonels' preferred battlefield coincide, ties occur on both battlefields; otherwise, each colonel wins his preferred battlefield.

${ }^{4}$ This idea is analogous to the idea of a pivotal vote in voting games.
} 
Lemma 1 Assume there is a pure strategy equilibrium $t^{*} \in \mathcal{T} \times \mathcal{T}$. When ties occur in (at least) one battlefield, only one troop is deployed in the battlefields that are won by either colonel. Formally, $\exists n, m: t_{n}^{* i}=t_{n}^{* j}$ and $t_{m}^{* i} \neq t_{m}^{* j}$ then $t_{m}^{* i}+t_{m}^{* j}=1$.

Proof. Whenever a colonel loses a battlefield, all troops he deploys in that battlefield are non-decisive. It thus follows that in any pure strategy equilibrium with (at least) one battlefield tied, such colonel should deploy 0 troops in the lost battlefield. In turn, the winning colonel should only deploy a single troop, should all his troops be decisive on the won battlefield.

We can now characterize the types of troop deployments that can be observed in battlefields that are tied.

Lemma 2 Assume there is a pure strategy equilibrium $t^{*} \in \mathcal{T} \times \mathcal{T}$. When positive ties occur in more than one battlefield, both colonels deploy a single troop in all battlefields where positive ties occur. Formally, $\exists n, m: t_{n}^{i}=t_{n}^{j}>0$ and $t_{m}^{i}=t_{m}^{j}>0$ then $t_{n}^{i}=t_{m}^{i}=$ 1.

Proof. We prove this Lemma by contradiction. Assume that there is an equilibrium with positive ties in two battlefields and strictly more than one troop in, say, battlefield $n$. That is, $t_{n}^{i}=t_{n}^{j} \geq 2$ and $t_{m}^{i}=t_{m}^{j}$. First note that all battlefields with positive ties should yield the same payoff; otherwise any colonel has a profitable deviation by diverting the troops from the least preferred battlefield to the most preferred battlefield.

Any colonel can deviate 2 troops from battlefield $n$ and obtain a higher payoff. The first troop can be deployed in battlefield $m$ by which the overall payoff does not change (instead of tying battlefields $n$ and $m$ now the colonel wins battlefield $m$ and loses battlefield $n$ ). The second troop can now be deployed in a territory that is tied or one that is lost (by Lemma 1 territories can only be lost by one vote) thus obtaining a strictly higher payoff.

The previous results fully characterize the equilibria when $T$ is small: few troops imply that a non-decisive vote can always be used for breaking a tie or reaching a tie in a battlefield lost 1-0 (i.e. the losing colonel deploys no troops and the winning colonel deploys a single troop). The previous results also imply that when $T$ is large there can never be an equilibrium with positive ties in more than one battlefield. This is because in all positive ties both colonels need to invest a single troop and battlefields that are not tied should only have a troop from one of the colonels. However, if $T$ is large enough, there are not enough battlefields where all troops can be deployed thus there should be a battlefield tied with a large number of troops. The following Lemma further characterizes the equilibrium voting profiles when $T$ is large. 
Lemma 3 When $T>\frac{N}{2}$, all pure strategy equilibria, $t^{*} \in \mathcal{T} \times \mathcal{T}$, have (at least) a battlefield with positive ties. When more than one positive tie occurs it should be the case that only one troop per colonel is deployed in each of the battlefields with positive ties.

Proof. The proof of the second statement is analogous to the one in Lemma 2. We now prove the first statement by contradiction: we assume that there is a pure strategy equilibrium without positive ties. Having more troops than half the battlefields $\left(T>\frac{N}{2}\right)$ implies that there are non-decisive votes. This implies that ties cannot occur in any battlefield and every battlefield needs to be won by one of the colonels. We now show that each colonel can win at least half of the battlefields (step 1) but such configuration implies that all battlefields are won with a single troop deployed in them (step 2). However, the latter assertion together with not having positive ties (inductive assumption) implies that the assumed equilibrium profile cannot constitute an equilibrium when $T>\frac{N}{2}$.

Step 1: Assume there is an equilibrium $\left(t^{1}, t^{2}\right)$ such that colonel 1 wins the battlefields indexed from 1 to $k$, and colonel 2 wins the remaining ones (indexed $k+1$ to $N$ ). Consider a situation where colonel 2 only deploys the necessary votes to win battlefields $k+1$ to $N$. This is, $\tilde{t}_{n}^{2}=t_{n}^{1}+1$, for $n=k+1, \ldots, N$. Colonel 1 needs a strictly higher number of troops than colonel 2 in battlefields 1 to $k$ so that $\tilde{t}^{2}$ is not a profitable deviation for colonel 2 , i.e. $t_{1}^{1}+\ldots+t_{k}^{1} \geq k+\left(\tilde{t}_{1}^{2}+\ldots+\tilde{t}_{k}^{2}\right)$. Using the definition of $\tilde{t}_{n}^{2}$ and the fact that any colonel disposes of $T$ troops, we know that the previous inequality can only be sustained when $k \leq \frac{N}{2}$. Therefore, if $\left(t^{1}, t^{2}\right)$ is an equilibrium it must be the case that none of the colonels wins more than $\frac{N}{2}$ battlefields. Whenever $N$ is odd, this proves our result: no colonel can win more than $\frac{N}{2}$ battlefields but this implies that there is a battlefield where ties occur and this contradicts our inductive hypothesis.

Step 2: Assume $\left(t^{1}, t^{2}\right)$ is a pure strategy equilibrium where both colonels win exactly half the battlefields when $N$ is even (w.l.o.g. assume colonel 1 wins battlefields 1 to $\frac{N}{2}$ ). The troops not deployed in battlefields $\frac{N}{2}+1$ to $N$ by colonel 2 need to be strictly smaller than the troops deployed by colonel 1 in any of the first $\frac{N}{2}$ battlefields. That is, $T-\left(t_{\frac{N}{2}+1}^{2}+\ldots+t_{N}^{2}\right)<t_{n}^{1}, \forall n=1, \ldots, \frac{N}{2}$. We can rewrite this expression as:

$$
t_{1}^{1}+\ldots+t_{\frac{N}{2}}^{1}<\frac{N}{2}+t_{n}^{1}, \forall n=1, \ldots, \frac{N}{2}
$$

The equality above implies that any sum of $(N-1)$ colonel 1's troops in the first half of the battlefields is strictly less than $\frac{N}{2}$. The fact that this very same colonel wins those battlefields implies that he should be deploying at least one troop in each of them -thus the sum needs to be equal to $\frac{N}{2}-1$. This implies that $t_{1}^{1}=\ldots=t_{\frac{N}{2}}^{1}=1$ but this is not an admissible configuration when $T>\frac{N}{2}$. 
The situation where more than one battlefield is tied requires colonels to be indifferent amongst battlefields where positive ties occur. Imagine for instance a situation with three battlefields that are equally valued by both colonels (i.e. $\theta_{1}^{i}=\theta_{2}^{i}=\theta_{3}^{i}$ for $i=1,2$ ). It can easily be shown that when $T=3$ the unique pure strategy equilibrium has each colonel deploying a single troop in each battlefield. However, both colonels deploying one troop each in the first battlefield and two troops each in the second battlefield does not constitute an equilibrium.

We can illustrate the above results following the previous example with 3 troops and battlefields $(N=T=3)$ but allowing any payoff per battlefield. The deployment $\left(t^{1}, t^{2}\right)=$ $((1,2,0),(0,1,2))$ can never constitute an equilibrium (regardless of the colonels' payoffs). This is because colonel 2 can profitably deviate by deploying one troop from the third battlefield into the first (or second) battlefield. The following list displays the only ten deployment profiles that can constitute a pure strategy equilibrium (note that we should have grouped all permutations of identical profiles)

- $\left(t^{1}, t^{2}\right)=((1,1,1),(1,1,1))$

- $\left(t^{1}, t^{2}\right)=((2,1,0) \cdot(2,0,1))$ or $((2,0,1) \cdot(2,1,0))$ or $((1,2,0) \cdot(0,2,1))$ or $((0,2,1) \cdot(1,2,0))$ or $((1,0,2) \cdot(0,2,1))$ or $((0,1,2) \cdot(1,2,0))$.

- $\left(t^{1}, t^{2}\right)=((3,0,0),(3,0,0))$ or $((0,3,0),(0,3,0))$ or $((0,0,3),(0,0,3))$

This example shows how the previous three Lemmas have greatly simplified the characterization of the games that have a pure strategy equilibrium in a game with three battlefields. ${ }^{5}$ As we increase the number of battlefields (and troops) the gains increase exponentially. We now need to show the payoff configurations that support such deployment of troops as a pure strategy Nash equilibrium.

\subsection{An algorithm to deploy troops}

We consider an algorithm that instructs colonels on how to allocate their $T$ troops sequentially. In each iteration of the algorithm, both colonels simultaneously deploy one troop in the battlefield they most value (amongst the battlefields each colonel is not winning).

This algorithm reaches a unique deployment profile when colonels are never indifferent among battlefields. However we need to add a couple of refinements to address the cases of indifference (these refinements will allow the reach of a deployment profile that is the unique pure strategy equilibrium when it exists). To illustrate these cases we consider

\footnotetext{
${ }^{5}$ The set of deployment profiles for both colonels contains 100 elements. The previous three Lemmas show that only 10 of them can constitute a pure strategy Nash equilibrium.
} 
once again a situation with three battlefields and three troops where the colonels' payoffs are: $t^{1}=(7,4,1)$ and $t^{2}=(2,5,5)$. Without having a rule, the algorithm may reach the deployment profile $(1,2,0)$ for one of teh colonels and $(0,1,2)$ for the other colonel -this profile is not an equilibrium. However, we could have also reached the deployment profile $\left(t^{1}, t^{2}\right)=((1,2,0),(0,2,1))$ that indeed constitutes a pure strategy equilibrium (below we will show that this is the unique equilibrium). The refinement that allows us to select the second deployment profile reads as follows: whenever a colonel reaches an iteration in which he is indifferent among various battlefields he deploys his troops in the battlefield least preferred by his opponent (among those to which he is indifferent). The second refinement helps the colonel to allocate his troop when the first refinement still leaves him indifferent among various battlefields: when there is not a single battlefield that is least preferred by his opponent (among those to which the colonel is indifferent) the colonel should deploy his troop in the battlefield where least troops have been deployed. This last requirement allows to evenly distribute troops when colonels are indifferent among many battlefields and allows them to reach the unique equilibrium when both colonels equally value all battlefields.

The previous algorithm (together with the two refinements) allows colonels to deploy all their troops. Moreover, the final deployment of troops is uniquely determined (except for some cases when there is indifference among battlefields). Most interestingly, the following proposition states that whenever there is a pure strategy equilibrium, the algorithm above reaches such allocation.

Proposition 1 Consider a non-zero Colonel Blotto game with a pure strategy equilibrium. The algorithm where each colonel simultaneously deploys a single troop at a time in the battlefield he values most among those that he is not winning (and in case of indifference, the battlefield that is least valued by his opponent and/or the battlefield in which there are less troops) reaches the pure strategy equilibrium's deployment profiles.

Proof. We first need to consider all deployment profiles that can be sustained as a pure strategy equilibrium (Lemma 1, 2, and 3) and show which payoff configurations can sustain such deployment profiles. Once this is done we can show that the described algorithm reaches such deployment configuration.

First, we consider the situation where there is a pure strategy equilibrium with all battlefields are won $1-0$ or tied $0-0$. These deployment profiles are an equilibrium only when the battlefield each colonel wins is valued strictly more than those he does not win. This implies the algorithm reaches exactly the same allocation as the pure strategy equilibrium.

Second, we consider the situation where there is a pure strategy equilibrium with at least two battlefields with positive ties and where the remaining battlefields that are won 
$1-0$ or tied $0-0$. We first note that a colonel's payoffs from the battlefields that are positively tied need to be equal. These payoffs need to be (strictly) greater than the payoffs from battlefields that are lost or tied $0-0$, and (strictly) lower than the payoffs from battlefields that are won. When the algorithm is run, colonels first allocate their troops into the battlefields they most prefer (i.e. battlefields whose outcome is $1-0$ ). At one point during the algorithm, each colonel has as many non-deployed troops as battlefields most valued and not won by any colonel; moreover, this set of battlefields yield the same payoff to each colonel. The algorithm's second refinement implies that ties with one troop occur in all these battlefields (i.e. $1-1$ ).

Third (and last), we consider the situation where there is a pure strategy equilibrium with a unique battlefield with positive ties, the remaining battlefields are won $1-0$ or tied $0-0$. Once again, the battlefields that are won are valued strictly more than those that are positively tied. And the latter should be valued more than the battlefields that are lost. The algorithm requires troops to be deployed in the battlefields that are most valued. The battlefield positively tied is valued strictly more than the lost battlefields, thus colonels continue to simultaneously deploy a single troop into that same battlefield because it is the most preferred among the battlefields each colonel is not winning.

We must recall that the previous result states that our algorithm reaches a pure strategy equilibrium when this one exists. It is easy to show that our algorithm does not always reach a pure strategy equilibrium. For instance, when payoffs are $\theta^{1}=(5,4,3)$ and $\theta^{2}=(7,3,2)$ the allocation reached by our algorithm has all troops of both colonels deployed in the first battlefield. However, the first colonel has a profitable deviation: he could deploy half his troops in battlefield 2 and the other half in battlefield 3. It follows from the previous Proposition that a game with such a payoff configuration cannot have a pure strategy equilibrium.

An interesting question that arises when analyzing our algorithm is whether the allocation it reaches constitutes a subgame perfect Nash equilibrium of the extensive game where each colonel simultaneously deploys a single troop in each stage of the game. The previous example answers this question negatively: our algorithm does not reach a subgame perfect equilibrium in the extensive game. However, when a pure strategy equilibrium exists in the simultaneous game, the algorithm reaches the unique subgame perfect equilibrium of game where each colonel repeatedly (and simultaneously) deploy a single troop.

Lemma 4 Consider a non-zero Colonel Blotto game with a pure strategy equilibrium. The algorithm where each colonel simultaneously deploys a single troop at a time in the battlefield he values most among the ones he is not winning (and in case of indifference, the one that is least valued by his opponent and/or has less troops deployed in it) is a subgame perfect equilibrium of the extensive form game with $T$ stages where each colonel simultaneously deploys a single troop in each stage of the game. 
Proof. We need to show that the algorithm prescribed actions indeed constitute a Nash equilibrium (NE) in all subgames of the extensive form game.

Using Proposition 1, we know that the deployment profile reached by the algorithm is a NE. Now we need to consider all other subgames. At any subgame or iteration of our algorithm we can drop the battlefields that have been won by any of the agents (when a pure strategy equilibrium exists, these battlefields play no role in the allocation of subsequent troops). By doing so we have a reduced colonel Blotto game with less battlefields; in addition, all remaining battlefields are tied. ${ }^{6}$ Using once again Proposition 1 we know that the algorithm reaches the unique equilibrium of the reduced game, thus the prescribed actions in our algorithm are indeed a NE in all subgames of our extensive game.

Whenever the game has non-pure strategy equilibrium the deployment profile reached by the algorithm is trivially non subgame perfect because it does not constitute an equilibrium of the game.

\subsection{Characterization of games with pure strategy equilibrium}

Proposition 1 tells us that a necessary condition for the existence of a pure strategy equilibrium is that the algorithm reaches an admissible deployment profile (see Lemmas 1, 2, and 3). This profile requires battlefields to be won by one troop or tied. Besides, in a pure strategy equilibrium a colonel that wins a battlefield should obtain a higher payoff from that battlefield than from the battlefields he ties. In turn, he should obtain a higher payoff from the battlefields he ties than from the ones he loses (strictly higher payoff when ties are with a positive number of troops). In order to simplify our analysis we assume that colonels are never indifferent between any two battlefields (including the possibility of indifference makes our analysis more tedious). When there is no indifference, we can prove that there can never be multiple pure strategy equilibria.

Lemma 5 Consider a non-zero Colonel Blotto game where colonels never receive the same payoff from any two battlefields (i.e. $\theta_{n}^{i} \neq \theta_{m}^{i}$ for any $n \neq m$ and for $i=1,2$ ). There can be at most a single pure strategy equilibrium.

Proof. Given a pure strategy equilibrium, Proposition 1 tells us that the equilibrium deployment profile is reached by our algorithm. Besides, our algorithm reaches a unique configuration when colonels are never indifferent between any two battlefields. It follows that if an equilibrium in pure strategies exists, it should be unique.

\footnotetext{
${ }^{6}$ It is possible that one of the battlefields may be positively tied. This happens when both colonels have invested their troops in the same battlefield in the previous iteration of the algorithm.
} 
In order to characterize the non-zero Colonel Blotto games that contain a (unique) pure strategy equilibrium it will be convenient to classify such games in terms of the coincidence of their most preferred battlefields. In this vein we first define the set of the $k(0<k<N)$ most preferred battlefields by colonel $i(i=1,2)$ as $M_{k}^{i}$. Formally this set can be described by the following expression:

$$
M_{k}^{i}:=\left\{n: \theta_{n}^{i} \geq \theta_{(k)}^{i}\right\}
$$

where $\theta_{(k)}^{i}$ denotes colonel i's $k$-th most preferred battlefield.

Recall that the algorithm above requires each colonel to distribute a single troop in his most preferred battlefield among the battlefields that he is not winning. This shows that as long as the most preferred battlefields of both colonels do not coincide, colonels place a troop in their most preferred battlefield and win it with the only permissible troop allocation in a pure strategy equilibrium (recall Lemma 2, battlefields can only be won $1-0$ ). When their most preferred battlefield (among those that they do not win) coincide, the algorithm leads to the remainder of their troops being deployed in such battlefield. The problem arises when one colonel's most preferred battlefield coincides with a battlefield that has already been won by his opponent. In such circumstances no pure strategy equilibrium will exist.

We say that a non-zero Colonel Blotto game has index $\lambda$ when $\lambda$ is the highest integer such that the sets of $\lambda$ most preferred battlefields by each colonel are disjoint, i.e. $\lambda=$ $\max \left\{k: M_{k}^{1} \cap M_{k}^{2}=\emptyset\right\}$ and $\lambda=0$ when $M_{1}^{1} \cap M_{1}^{2} \neq \emptyset$. The index of any game is always well defined: greater or equal than 0 and smaller or equal than the integer value of $\frac{N}{2}$. For instance, a game with index equal to 0 is one where both colonels' most preferred battlefield coincides; a game with an index equal to 1 is one where both colonels' most preferred battlefields do not coincide but their second most preferred battlefields coincides with each other or with the most preferred of their opponent, etc.

With the aid of the index of non-zero Colonel Blotto games we can characterize the deployments achieved by our algorithm. This in turn allows to characterize the games that have a pure strategy equilibrium. Prior to the statement of our main Proposition we present two examples that perfectly capture the situations when a pure strategy equilibrium exists.

Example 1 Consider a situation where both colonels' payoffs are: $(4,5,3)$ and $(9,2,1)$. If both colonels only have single troop $(T=1)$ there is indeed an equilibrium where both colonels deploy that troop in their most preferred battlefield (note that non-zero sum colonel Blotto games with a single troop $(T=1)$ always have a pure strategy equilibrium).

Example 2 Now consider a situation where both colonels payoffs are: $(5,4,3)$ and $(9,2,1)$. If both colonels have 3 troops, we have that our algorithm reaches a deployment profile 
where all troops are deployed in the first battlefield. However, we can see that this does not constitute an equilibrium because the first colonel has incentives to divert his troops into the last two territories. Instead, if his valuation of the last two territories is low enough, the described deployment profile would be an equilibrium.

As shown in example 1, there always exists an equilibrium whenever $T=1$. We are now ready to state our Proposition that characterizes the valuations of the games which have a pure strategy Nash equilibrium whenever $T>1$.

Proposition 2 Consider a non-zero Colonel Blotto game with $T>1$ troops, $N$ battlefields and index $\lambda \geq 0$. Assume that colonels never receive the same payoff from any two battlefields. When the number of troops is smaller than or equal to the index $(T \leq k)$, there is a unique pure strategy equilibrium. When the number of troops is greater than the index $(T>k)$ there exists a pure strategy equilibrium, if and only if both colonels $(\lambda+1)$ most preferred battlefield coincides and the colonels' valuation of this battlefield is large enough.

Proof. When there is a small number of troops $(T \leq \lambda)$ our algorithm reaches an allocation in which he wins his $\lambda$ preferred battlefields, loses $\lambda$ other battlefields and ties the remaining ones. This deployment profile is indeed an equilibrium because each troop is deployed in the $T$ battlefields that yield the most payoff to each colonel.

When there is a large number of troops $(T>\lambda)$ the existence of pure strategy equilibrium depends on the $(\lambda+1)$ most preferred battlefield of each colonel, $\theta_{(k+1)}^{i}$ for $i=1,2$. When both colonels $(\lambda+1)$ most preferred battlefield coincides, the algorithm reaches a deployment profile in which each colonel deploys a single troop in his $\lambda$ preferred battlefields and $(T-\lambda)$ troops in his $(\lambda+1)$ most preferred battlefield. The outcome of such a battle has each colonel winning his $\lambda$ most preferred battlefields, tying his $(\lambda+1)$ most preferred battlefield, losing $\lambda$ other battlefields and tying the remainder. This may be a candidate to pure strategy equilibrium as the deployment profile satisfies the conditions in Lemmas 1, 2, and 3. In order to ensure this is an equilibrium we need to check that there is no profitable deviation. We know that each colonel is winning his $\lambda$ most preferred battlefields with a single troop so there is no incentive to move troops in or out of those battlefields. The question is whether a colonel is better off by relocating the $(T-\lambda)$ troops deployed in his $(\lambda+1)$ most preferred battlefield. These troops can be relocated to improve the outcome of one of the territories that he ties or loses. We are now ready to show that there is a lower bound in $\theta_{(\lambda+1)}^{i}$ above which the actions described constitute an equilibrium.

Colonel $i$ 's deployment profile is such that apart from his $(\lambda+1)$ most preferred battlefields, all battlefields are either lost by one troop or tied. In the former case, two troops are 
needed to win such territory and in the latter, a single troop suffices. In order to explicitly describe the lower bound for a pure strategy equilibrium to exist we define the function $\rho^{i}(n)$ as

$$
\rho^{i}(n)=\left\{\begin{array}{l}
1 \text { when the } n \text {-th most preferred battlefield of colonel } i \text { is tied } \\
2 \text { when the } n \text {-th most preferred battlefield of colonel } i \text { is lost. }
\end{array}\right.
$$

We know that colonel $i$ has a profitable deviation when there exists $\widetilde{\rho} \in\{1,2\}$ and $\nu \in\{1, \ldots, N\}: \widetilde{\rho} \leq \rho(\lambda+\nu)$ and

$$
\begin{aligned}
& \theta_{(\lambda+1)}^{i} \leq \rho^{i}(\lambda+2) \cdot \theta_{(\lambda+2)}^{i}+\ldots+\rho^{i}(\lambda+\nu-1) \cdot \theta_{(\lambda+\nu-1)}^{i}+\widetilde{\rho} \cdot \theta_{(\lambda+\nu)}^{i} \\
& \text { where } \nu \text { is such that } \rho^{i}(\lambda+2)+\ldots+\rho^{i}(\lambda+\nu-1)+\widetilde{\rho} \leq T-\lambda .
\end{aligned}
$$

In other words, the above formula applies when colonel $i$ can relocate (some or all) of his troops in his $(\lambda+1)$ most preferred battlefield into his next $\nu$ most preferred battlefields. This relocation of troops implies that he loses his $(\lambda+1)$ most preferred battlefield but wins $\nu-1$ battlefields (from being tied or lost) and improves the outcome of his $(\lambda+\nu)$ most preferred battlefield (colonel $i$ may not have enough resources to win this last battlefield).

Note that the lower bound on $\theta_{(\lambda+1)}^{i}$ not only depends on the number of troops available (the colonel is relocating at most the $(T-\lambda)$ troops deployed in his $(\lambda+1)$ most preferred battlefield) but also on the particular identity of the $\lambda$ most preferred battlefields by his opponent.

We now need to look at the case where the colonels' $(\lambda+1)$ most preferred battlefield does not coincide. By the definition of the index of the game we know that $M_{\lambda+1}^{1} \cap M_{\lambda+1}^{2} \neq \emptyset$. It could be the case that both colonels' $(\lambda+1)$ most preferred battlefield coincides with one the $\lambda$ most preferred battlefields of his opponent (i.e. $\theta_{(k+1)}^{i} \in M_{k}^{j}, \forall i \neq j$ ), or that this occurs only for one of the colonels.

In the first case we have that $\theta_{(k+1)}^{i} \in M_{k}^{j}, \forall i \neq j$. The deployment reached by our algorithm now depends on whether $(T-\lambda)$ is even or odd. Note that at the stage when colonel $i$ is deploying his $(\lambda+1)$ troop, he ties a battlefield he was losing (one of the $\lambda$ most preferred by his opponent) and the deployment of his opponent implies that a battlefield he was previously winning is now tied. At the stage when colonel $i$ is deploying his $(\lambda+2)$ he wants to deploy the troop in the battlefield that is most preferred to him among the battlefields that he is not winning. This implies that he will undo the tie just created by the $(\lambda+1)$ troop of his opponent. His opponent will do exactly the same, thus the outcome of this deployment of troops will be identical to the outcome they obtained after allocating $\lambda$ troops. It thus follows that the following deployment of troops will simply replicate the outcome of stage $(\lambda+1)$, and the subsequent deployment will replicate that of stage $(\lambda+2)$. Therefore, when $(T-\lambda)$ is odd, the algorithm ends in the outcome achieved in the $(\lambda+1)$ stage, and when it is even, it ends in the outcome 
achieved in the $(\lambda+2)$ stage. Finally, we need to show which of these situations can constitute a pure strategy Nash equilibrium: the allocation where $(T-\lambda)$ is even is one where two battlefields are won with strictly more than 1 troop and we know that this cannot constitute an equilibrium (Lemma 1); the allocation when $(T-\lambda)$ is odd cannot constitute an equilibrium because two battlefields are tied with one troop per colonel but, given that colonels are not indifferent between any two battlefield, there is a profitable deviation by deploying the troop in the tied battlefield that yields less payoff into the tied territory that yields more payoff.

In the second case we have that $\theta_{(k+1)}^{i} \in M_{k}^{j}$ but $\theta_{(k+1)}^{j} \notin M_{k}^{i}$. The deployment profile reached by our algorithm implies that colonel $i$ wins all battlefields in $M_{\lambda}^{i}$, colonel $j$ wins battlefields in $M_{\lambda+1}^{j} \backslash M_{\lambda+1}^{i}$ and they both deploy $(T-\lambda)$ in colonel $i$ 's $(\lambda+1)$ most preferred battlefield. It is immediate to show that this cannot constitute an equilibrium because colonel $j$ has incentives to deviate the troop deployed in his $(\lambda+1)$ most preferred battlefield into his opponent's $(\lambda+1)$ most preferred battlefield: in this way he improves his overall payoff by tying a battlefield he is winning (his $(\lambda+1)$ most preferred battlefield) and wins a battlefield he is tying (on of his $\lambda$ most preferred battlefields).

An immediate corollary follows from the previous proposition.

Corollary 1 Consider a non-zero Colonel Blotto game with $T$ troops and $N$ battlefields. Assume that the payoffs to each colonel are independent and identically distributed according to a density with full support on $[0,1]$. There is a strictly positive probability that the game has a pure strategy equilibrium.

The proof is immediate because when payoffs are i.i.d. there is for instance a strictly positive probability that both colonels equally rank all battlefields and that the first battlefield's payoff to each colonel is arbitrarily larger than the payoffs of the other battlefields. In such circumstances a pure strategy equilibrium trivially exists. More noteworthy of highlighting is the probability with which pure strategy equilibria exists increases rapidly as we increase the number of battlefields (it is easier to find a situation where the sets of $T$ most preferred battlefields of each colonels are disjoint) but this probability will decrease rapidly as we increase the number of troops (it is easier to find a situation where both colonels' $(\lambda+1)$ most preferred battlefield coincide).

\section{Conclusion}

We have characterized the situations under which non-zero sum colonel Blotto games have pure strategy equilibria. We have done so in three steps. First, we have determined the admissable actions (deployment profiles). Second, we have introduced an algorithm that 
converges to a pure strategy equilibrium when this one exists. And third, we have characterized the set of payoffs that support pure strategy equilibria. Finally we have stated that when payoffs are independent and identically distributed there is always a positive probability of finding non-zero sum colonel Blotto games with pure strategy equilibria. We believe that this work only constitutes a first step towards the full characterization of equilibria in non-zero sum colonel Blotto games. These games are not only relevant in terms of conflict games but can also be of use when thinking about the allocation of resources in voting games, optimal strategies in multi-object auctions, etc.

\section{References}

[1] Borel, E. (1921), "La theorie du jeu les equations integrales a noyau symetrique", Comptes Rendus de l'Academie 173; English translation by Savage, L (1953), "The theory of play and integral equations with skew symmetric kernels", Econometrica, 21.

[2] Borel, E. and J. Ville (1938), "Application de la The orie des Probabilite s aux Jeux de Hasard," Gauthier-Villars, Paris, 1938. [Reprinted at the end of E. Borel and A. Che ron, "Theorie mathe matique du bridge a la porte e de tous," Editions Jacques Gabay, Paris, 1991].

[3] Casella, A. (2005), "Storable Votes", Games and Economic Behaviour, 51.

[4] Gross, O., Wagner, R. (1950), "A continuous Colonel Blotto game", RAND Corporation RM-408.

[5] Hart, S. (2008), "Discrete Colonel Blotto and General Lotto Games", International Journal of Game Theory, Vol 36

[6] Hortala-Vallve, R. (2007), "Qualitative Voting", Department of Economics Discussion Papers Series (Oxford University), No 320

[7] Jackson, M.O. and H.F. Sonnenschein (2007), "Overcoming Incentive Constraints by Linking Decisions", Econometrica, 75, 241-257.

[8] Kvasov, D. (2007), "Contests with limited resources", Journal of Economic Theory, 136.

[9] Roberson, B. (2006), "The Colonel Blotto Game", Economic Theory, 29

[10] Roberson, B. and Kvasov, D. (2008), "The Non-Constant-Sum Colonel Blotto Game" (August 2008). CESifo Working Paper Series No. 2378. 